\title{
A Review on Gold Nanoparticles-Based Biosensors in Clinical and Non- Clinical Applications
}

\author{
Nika Momeni, Kayla Javadifar, Maria A. Patrick, Muhammad H. Hasan and Farhana M. \\ Chowdhury
}

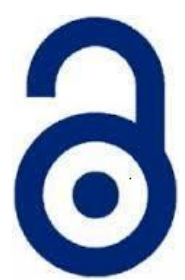

Received: 13 August 2021

Accepted: 24 November 2021

Published: 01 January 2022

Publisher: Deer Hill Publications

(c) 2022 The Author(s)

Creative Commons: CC BY 4.0

\begin{abstract}
Gold nanoparticles (GNP) acquire unique properties that have made significant contributions to clinical and nonclinical fields, specifically in the application of GNP's for designing biosensor devices in which exhibit novel functional properties. Many properties of GNP's are reviewed in this literature including optical properties, biocompatibility, conductivity, catalytic properties, high surface-to-volume ratio, and high density of the GNPs, that make them excellent in the application of constructing GNP-based biosensors. This literature review covers a specific comparison between the optical, electrochemical, and piezoelectric biosensors, as these are the three most common GNP-based biosensors. Optical biosensors are optimal due to their ability to cater to surface modification, which then leads to the ability for selective bonding. Furthermore, with the use of GNP and the sensor's non-invasive and non-toxic method of use, high-resolution images and signals can be formed. The sensitivity and specificity of electrochemical biosensors with the conductivity of GNPs, the electrodes of this stable biosensor can detect tumour markers in the human body. Piezoelectric biosensors are mass sensitive sensors and with the use of GNP, it amplifies the changes in mass. Through this, these sensors progress to be immunosensors which determine microorganisms and macromolecular compounds. As well, this review will conclude with an outline of present and future research recommendations for real-world application of the three GNP-based biosensors discussed.
\end{abstract}

Keywords: Gold nanoparticles, biosensors, sensitivity, selectivity

\section{INTRODUCTION}

In Canada, one of the leading causes of high mortality rates for many people is the delay in recognizing symptoms of diseases and seeking necessary medical attention. For certain diseases prevalent in the current day, the longer the patient waits to seek medical attention, the more fatal the consequences are to the patient, their friends, family, and the economy [1]. One of the primary reasons for these consequences is due to the lack of proper health monitoring of the patients during these wait times [1]. The delay in healthcare monitoring has economic consequences such as reduced productivity, reduced ability to work caused by untreated medical conditions, increased absenteeism. As well, the advance of disease during a delay in patient health monitoring can mean having to undergo longer, more complex and intensive treatments than would be required if the problem symptoms were identified sooner. Thus, leading to a poorer medical outcome. Over 15 years from 1994-2009, changes in wait times for cardiovascular care are associated with approximately 662 potentially avoidable deaths, if patients were monitored during their wait times [1]. Through this research and better implementation of cardiovascular care, patients' cardiovascular health has been increasingly monitored during wait times using a plethora of biosensing devices (e.g. halter monitors, etc) before cardiovascular surgery, resulting in a slow reduction in avoidable mortality. Cardiovascular health monitoring is only one of many applicable examples of how biosensing devices show promising change for patients, healthcare, and the economy [2]. Biosensors have a wide use in clinical and non-clinical applications; they are able to be used for the detection of diseases, diagnosis, treatment, patient health monitoring, and human health management. The early-

N. Momeni ${ }^{1}$ 凶, K. Javadifar', M. A. Patrick1, M. H. Hasan ${ }^{1}$ and F. M. Chowdhury ${ }^{2}$ 'Department of Electrical, Computer and Biomedical Engineering

Faculty of Engineering and Architectural Sciences

M5B2K3, Ryerson University, Canada

2Formerly Department of Community Medicine

Marks Medical College, Dhaka, Bangladesh.

E-mail: nika.momeni@ryerson.ca

Reference: Momeni, et al. (2022). A Review on Gold Nanoparticles-Based Biosensors in Clinical and Non-Clinical Applications. International Journal of Engineering Materials and Manufacture, 7(1), 1-12. 
stage detection and diagnosis of disease, with a treatment plan to combat the disease and monitoring of the patient's help, has proven to be the most proactive way to manage the life of a patient [2].

To process early-stage detection, the use of functionality must have properties that are highly sensitive to detect minuscule differences and that are highly responsive. This can be achieved through the use of biosensors. A biosensor is a device that is used for analyzing biological samples which helps to find biomarkers in the sample provided [3]. Through the sensor, one can see molecules such as proteins, DNA, and RNA that are expressed in excess or mutilated [4]. This sensor has the ability to convert chemical, biological, or biochemical responses into electrical signals [5]. The sensors are able to sense biological structures such as cells, and nucleic acids but also have the ability to measure the biological process and any physical changes to the patient [6]. Essentially, biosensors consist of 3 essential components where a bioreceptor reads the sample that is provided, which is then passed to the transducer which will be inputted into the electronic unit system which consists of the amplifier, processor, and display.

In pairing with the biosensors, various metal nanoparticles and nano dimensional conducting polymers have been used in biosensors [3]. More favourably, gold nanoparticles (GNPs) are used in laboratories due to their unique properties, and the roles in which they play in different sensing systems [3]. GNPs are favourable because of their unique optical properties and ease of use with various biomarkers in aqueous solutions [3]. Additionally, GNPs have a more stable immobilization of large amounts of biomolecules retaining their bioactivity and they permit fast and direct electron transfer between a variety of electroactive species and materials [3]. Furthermore, the light-scattering properties and extremely large enhancement ability of the local electromagnetic field enables GNPs to be used as signal amplification tags in diverse biosensors [3].

In this paper, three types of gold nanoparticles used in biosensors which are optical, electrochemical, and piezoelectric will be compared. Furthermore, there will be an analysis on which type of gold nanoparticles is most suitable for different applications. Moreover, there is a discussion on the advantages, disadvantages, and limitations each of the gold nanoparticles has for various applications and how it can be applied to be used to the best of its abilities. According to the three types of biosensors, the GNP- based biosensors are discussed separately with images showcasing the characteristics of each sensor.

\section{Gold-Nanoparticle (GNP) Biosensors}

Biosensors are increasingly integrating into clinical and non-clinical applications [7]. Their general composition consists of a functional bioreceptor, transducer, and an analog-to-digital (A/D) converter [8]. Bioreceptors are materials that interact with a bioanalyte, where the transducer transforms a signal that is produced by this interaction into a signal that can be quantified and analyzed, as seen in figure 1 [8]. To generalize, biosensors analyze specific, quantitative information using a bioreceptor.

Figure 1 displays how a signal is produced when a biological element such as an enzyme, DNA, or an antibody, recognizes the analyte it is trying to combine with. Examples of an analyte are a substrate, complementing DNA, or antigen. The bioreceptor must be sensitive to the target analyte to generate a signal, as clearly shown in figure 1 [8]. Applications of biosensors stem anywhere from pathology to pollution monitoring, as seen in figure 2 [9].

As of recently, gold nanoparticles (GNPs) have been applied to the production of biosensors. Due to their unique properties, they show great potential for the development of these sensors to produce accurate analytics, especially on a molecular scale [10]. GNPs increase the sensitivity, reliability, and selectivity of the biosensing process, however, although GNP-based biosensors are rapidly integrating into different sectors, they are still in the early stages of being used in real-world applications [10]. In general, there are three different types of biosensors: optical, electrochemical, and piezo-electric [11]. This journal explores these three types and the application of GNPs to these biosensors, and the studies are done to examine their effectiveness.

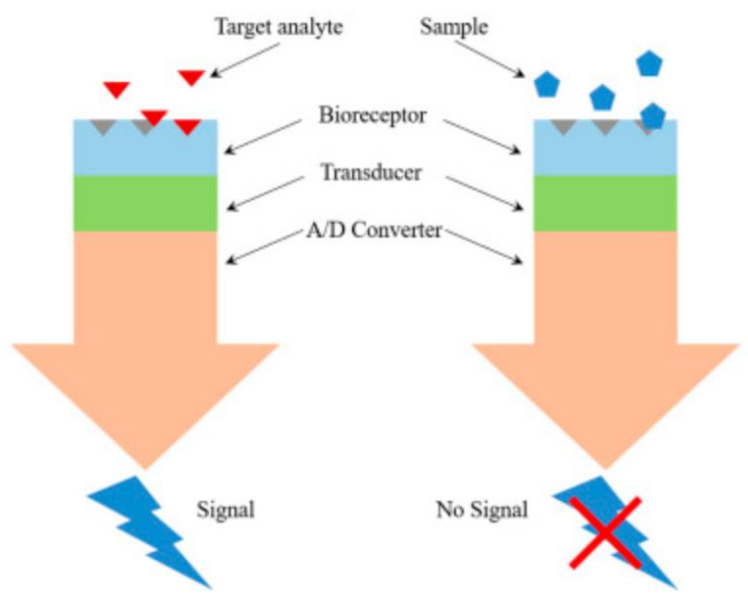

Figure 1: Visual representation of functionality of biosensors and how they produce a signal [8]. 


\section{OPTICAL BIOSENSORS}

To put it simply, optical sensors measure the changes in light (photons) [3]. They are the most common type of biosensor and interact with many biological materials, such as enzymes, antibodies, antigens, receptors, or cells/tissues, to produce a signal. The signal they produce is proportional to the concentration of the measured analyte (examples listed above) [12]. Figure 3 provides a visual representation of the composition of optical biosensors. It shows that they are analytical devices consisting of a biorecognition sensing element fused with an optical transducer system [12].

\subsection{Functionality}

GNP-based optical biosensors issue a spectrum of possibilities due to the collective oscillation of conduction of band electrons known as plasmons. These oscillations occur as a result of electromagnetic radiation applied externally [13]. Of all the optical transducers of these biosensors, the surface plasmon resonance (SPR) modality is an attractive choice for experimental investigation because of the interaction between electromagnetic waves and the conduction electrons in metals (in this case gold). SPRs are used in the classification of physiochemical changes of thin films on a metal surface, as seen in figure 4 [3].

Molecules of the target analyte bind to the surface of the metal film, causing change in the dielectric constant. This also leads to a change in the reflection as a result of the laser and the metal-liquid surface [3]. It is important to note that the gold-nanoparticles (GNPs) contain an extremely sensitive dielectric constant due to the gold material, adding to the list of reasons why GNPs have been investigated in SPRs for more accurate analyses [3].

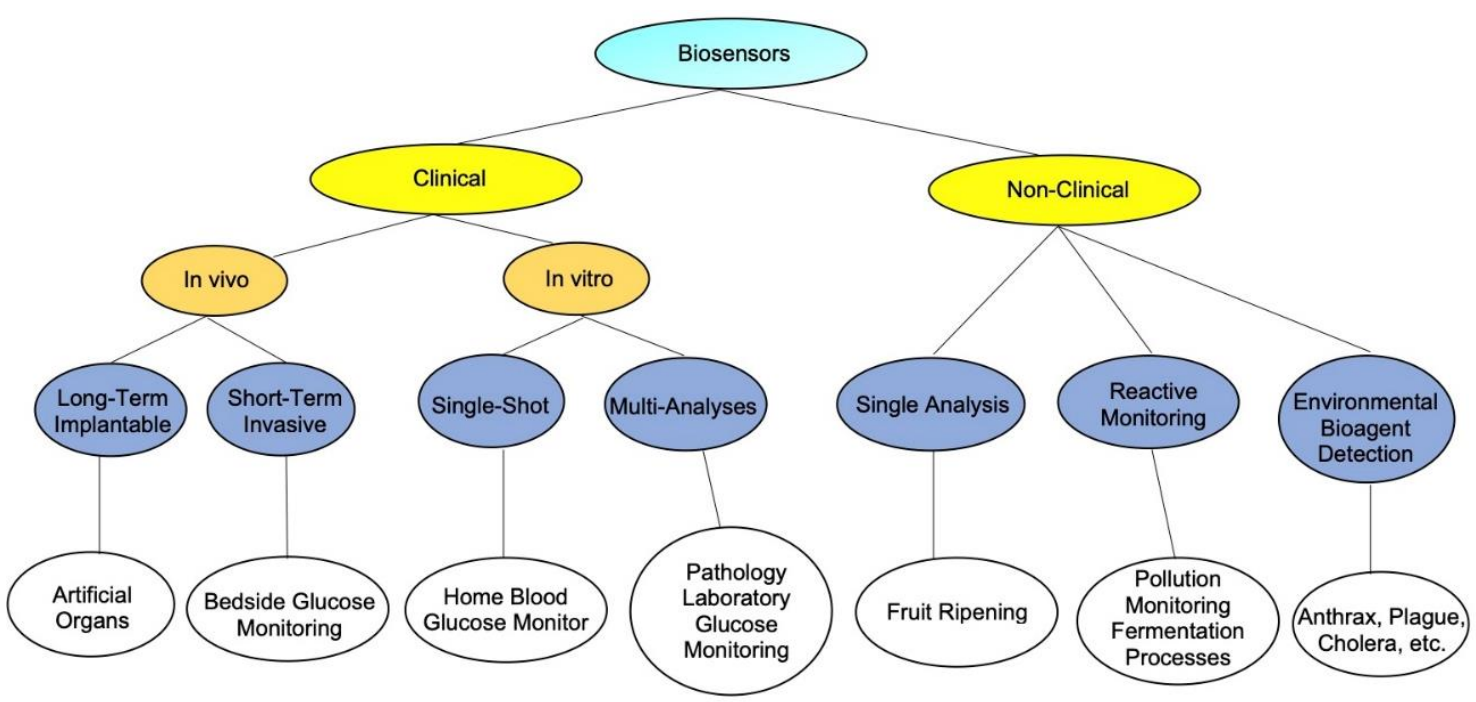

Figure 2: Applications of Biosensors and the different sectors they are used in [9].

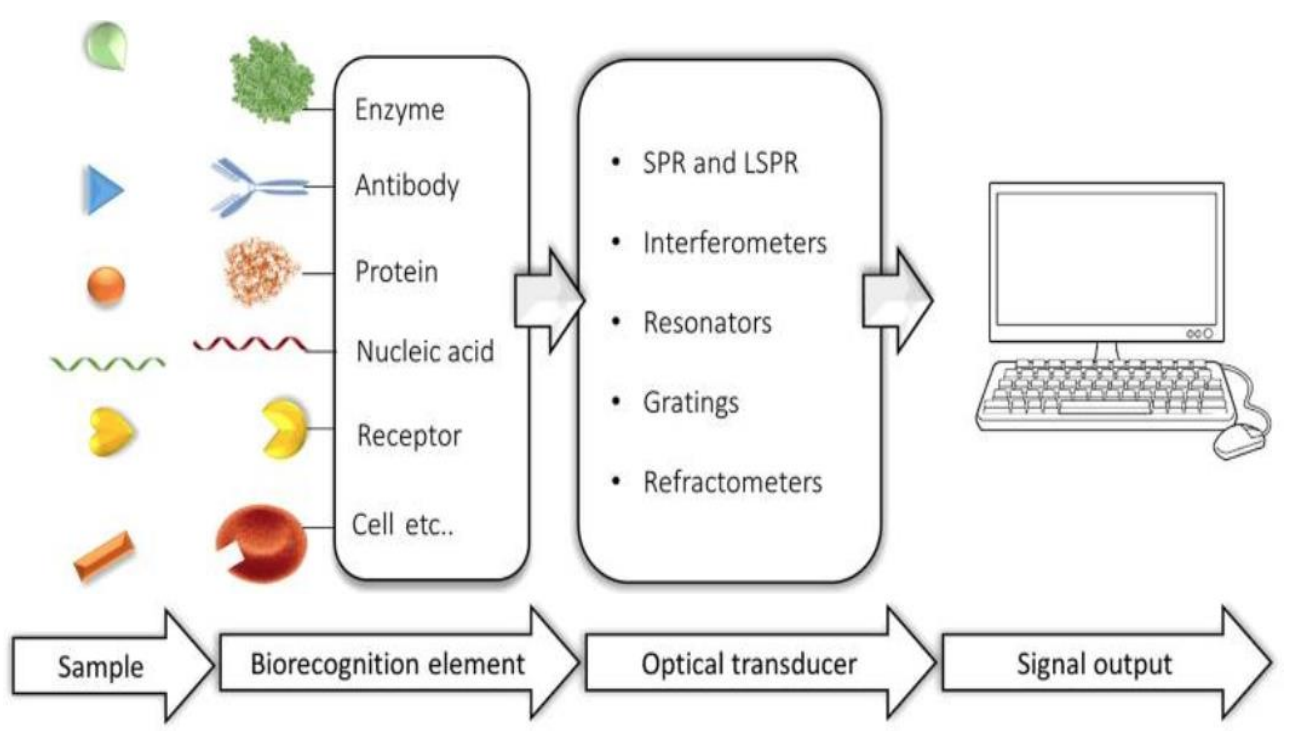

Figure 3: Flow chart of optical biosensor composition and how a signal is produced [12]. 


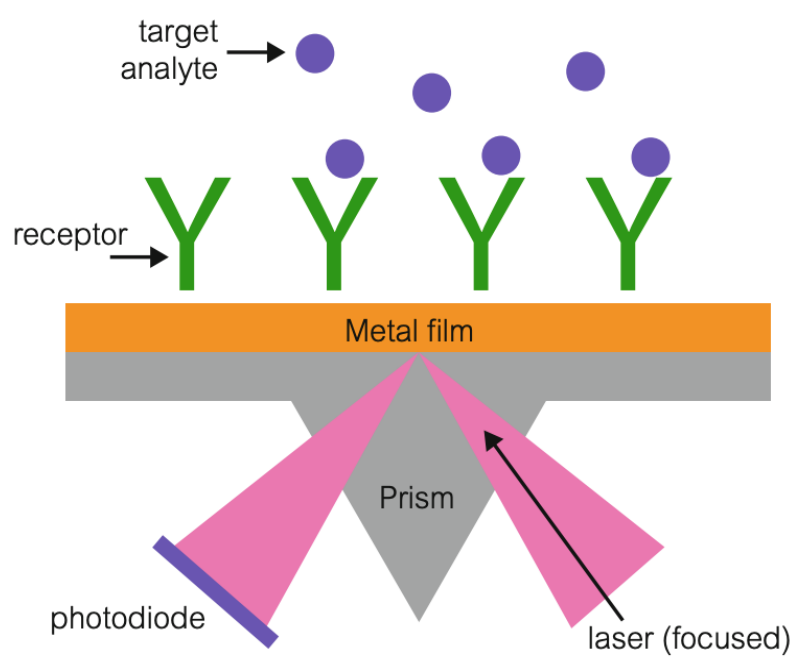

Figure 4: Diagram of the oscillation from excited electrons from electromagnetic radiation(resonance).

\subsection{Properties of GNPs in Optical Biosensors}

As mentioned, gold-nanoparticles display a range of optical properties that are explained by plasmonic effects, hence the desirability of the surface plasmon resonance (SPR) technique. The optical properties of GNPs are capable of altering their size, shape, composition, and coupling with other plasmonic nanoparticles in order to display the intensity of colour under light [14]. Gold-nanoparticles are also able to amplify other optical signals, which is key in biosensor development [14].

In addition to their optical properties, GNPs are advantageous because of their biocompatibility, inertness, easy use, and intense colours [14]. The specific property that GNPs possess that cater to the development of biosensors is surface modification. Through a variety of biomolecules, such as proteins, the development of biosensor platforms is possible. This is accomplished as the biomolecules form stable bonds leading to the creation of selective bonding [14]. Furthermore, there are a variety of biosensing practices (localized surface plasmon (LSPR), surface-enhanced Raman scattering (SERS), fluorescence, etc), but this section focuses specifically on surface plasmon resonance. Surface plasmon resonance can be modified into different sizes, shapes, and surface coupling among the nanoparticles, as mentioned above. This is achieved through the excitation of free electrons within gold-nanoparticles once irradiated with light. Following non-propagating oscillations of SPR. Gold surface plasmons are also greatly absorbent (with light) and contain a strong scattering of light; this property makes GNPs a focal point in the development of biological sensing and imaging probes [14].

\subsection{Applications: Optical Biosensors in Colorimetric Bioassays}

Optical biosensors are emerging in the biomedical engineering field at a rapid pace. Generally, optical biosensing/imaging is a non-invasive and non-toxic method in developing high-resolution images and signals. Through the integration of gold-nanoparticles, these output responses have become significantly more sensitive, specific, contrast, and multi exhibitable. Over the development of GNP optical biosensors, scientists have developed a spectrum of gold nanostructures of which each contains its range of optical properties, such as nanospheres, nanorods, and nanoshells, to name a few [15]. Figure 5 displays the developing applications of GNP-optical biosensors and the three methods (LSPR, SERS, and fluorescence) mentioned above with respect to optical biosensing and optical bioimaging [15].

Focusing on LSPR optical biosensing, colorimetric bioassays are a successful example of these devices. They function as a result of wavelength shift from changes in interparticle distance. Through GNPs, the selectivity and the sensitivity of the device improve leading to greater control of interparticle forces. Another advantage to this device is its simplicity in digital monitoring. The assays can be used with smartphone imaging for convenience and nearpatient testing platforms. Figure 6 provides an example of a microfluidic colorimetric bioassay used in E. coli detection [15]. The device is composed of 2 mixing channels: one for separation and the other for detection, the device is based on horseradish peroxidase (HRP), hydrogen peroxide, and tyramine. Once the aggregation process has been undergone, the size of the GNPs exponentially grows (in terms of wavelength) from $\sim 13 \mathrm{~nm}$ to $\sim 670 \mathrm{~nm}$. This colour change is detected through a smartphone app. In conclusion, GNPs will continue to integrate into optical biosensing/imaging techniques due to their non-invasive nature, specificity, high sensitivity, and reliability to provide/conduct accurate analyses. 


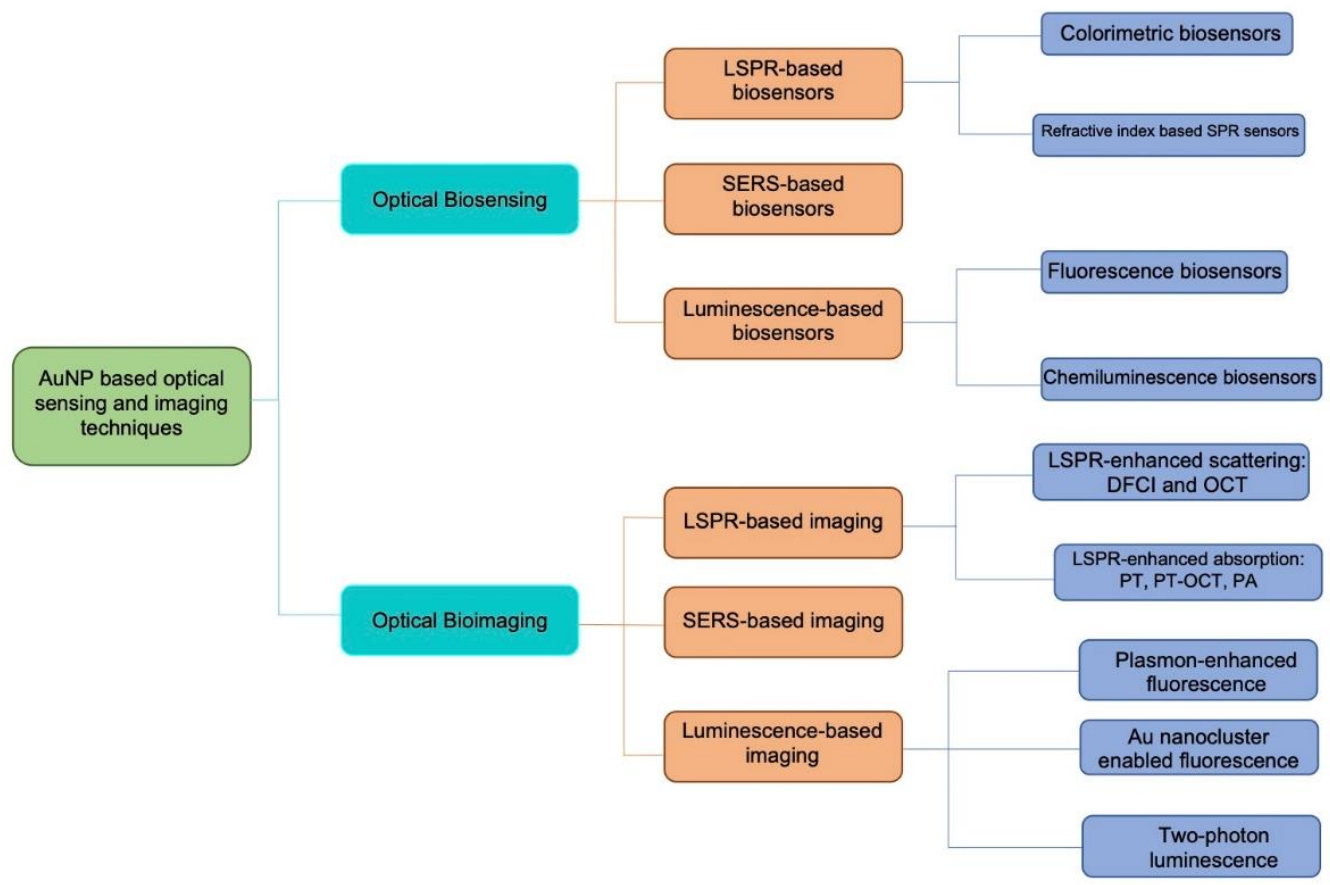

Figure 5: Applications of optical biosensing/bioimaging based on GNPs [15].
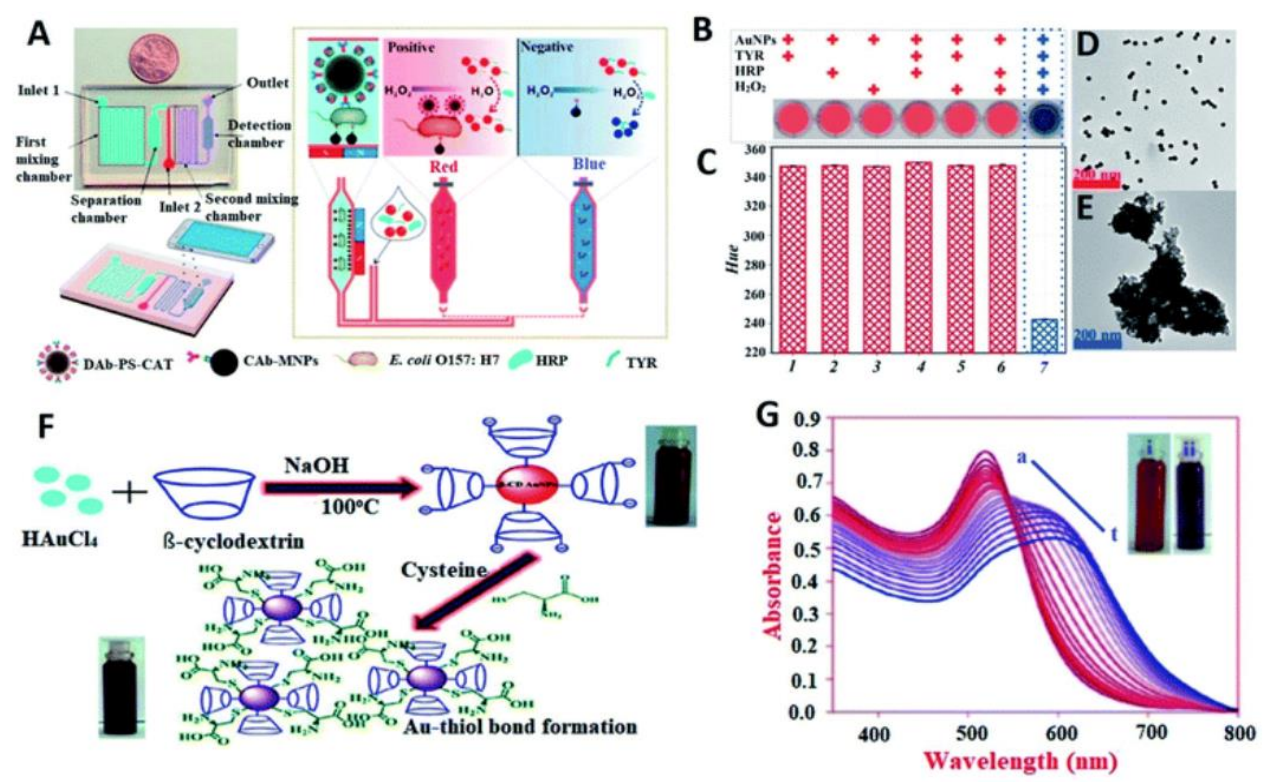

Figure 6: Smartphone based GNP optical biosensor for colorimetric assays for E. coli. (A) Labelled diagram of the biosensor; (B) result of mixing GNPs with various cross-linking agents; (C) Hues of colour changed; (D) TEM image before aggregation; (E) TEM image after aggregation; (F) Schematic of functionality of biosensor; ( $G$ ) SPR band changes [15].

\section{ELECTROCHEMICAL BIOSENSORS}

Electrochemical biosensors produce useful signals as a result of the conversion of biological binding events into these signals. They provide fast, simple, and low-cost detection properties. Additionally, the biocompatibility, conductivity, and catalytic properties make these types of biosensors, specifically GNP electrochemical biosensors, desirable for further research [3].

\subsection{Functionality}

In electrochemical biosensors, GNPs play many possible roles including electron wires, an immobilization platform, and an electrocatalyst. Beginning with GNPs as electron wires (electron transfer), the fundamental concept of this role revolves around bioelectrochemical reactions, in other words, redox reactions. The electron transfer that occurs 
between the electrode surface and the redox protein is the main reaction under scrutiny. A fallback of the interaction between electrodes and the active centres of oxidoreductases in the surrounding thick insulating protein that encompasses these active centres. This protein shell blocks the electron centre for these reactions, which results in a lack of analytics. However, the conductivity of GNPs enhances the electron transfer, regardless of the protein shell, and so the GNPs act as "electron wires" since they connect and improve the interaction between active centres of proteins and electrodes of the biosensor.

Electrochemical biosensors function based on bioelectrochemistry. Specifically, the bioactivity, stability, and quantity of biological recognition elements that are immobilized on the electrodes of these sensors. This process usually results in denaturation and loss of bioactivity in the absorption of biomolecules that are placed directly on that surface of bulk materials. The benefits of GNPs to resolve these losses are their absorption properties. The absorption of biomolecules on the surface of GNPs leads to the maintenance of bioactivity and stability as a result of the biocompatibility of GNPs and their high surface free energy. As mentioned in varying sections of this review, GNPs contain a higher surface area than flat gold surfaces. This allows for a larger protein loading and greater sensitivity.

The final role that GNPs play in electrochemical biosensors is an electrocatalyst. Although gold is chemically inert, GNPs showcase great promise for catalytic activity. These catalytic properties stem from the GNP's quantum scale dimension, large surface-volume ratio, and interface-dominated properties. These qualities are capable of decreasing overpotentials of electrochemical reactions, reverse some redox reactions, and could potentially allow the fabrication of enzyme-free biosensors. It should be noted that the properties listed of each role of GNPs in electrochemical biosensors will be explained in the next section, and that quantum scale dimension is beyond the scope of this review and will not be discussed herein.

\subsection{Properties of GNPs in Electrochemical Biosensors}

As seen in section 3.1, there are many desirable properties of GNPs used for each aspect of electrochemical biosensors. To list the ones above, conductivity, biocompatibility, high surface free energy, sensitivity, selectivity, and high surface-volume ratio.

The conductivity of GNPs allows and enhances electrochemical reactions to take place, which is essential to the fundamentals of electrochemical biosensors. Biocompatibility and the high surface free energy of these special nanoparticles allow for absorption to take place in these sensors to maintain the bioactivity and their stability. The sensitivity and selectiveness allow for selective electrochemical analysis as well as their high surface area that leads to improved sensitivity of these devices. Finally, the high surface-volume ratio is among the many properties that cause catalytic behaviours in GNPs. These GNP properties advance the electrochemical analytical characteristics and overall enable this biosensor to play many important roles.

\subsection{Applications: Electrochemical Biosensors in Clinical Diagnosis}

Electrochemical biosensors are used to detect tumour markers in the human body. Focusing specifically on prostate cancer biomarkers known as prostate-specific antigen, or PSA [16]. The electrodes of the electrochemical biosensor used in this biomarker detection are composed of gold nanoparticles/graphene oxide, anti-Total PSA monoclonal antibody, and anti-Free PSA antibody. It became evident that this biosensor possesses a high sensitivity toward total and free PSA, as well as high selectivity for PSA in comparison to other tumour markers. Electrochemical biosensors are desirable devices for detecting tumour markers due to their simplicity, fast response, and miniaturization, and the use of GNPs has added to this desirability to improve the sensitivity of these biosensors [16]. Additionally, since GNPs are inert/stable, biocompatibility/less hazardous, and simple, they can enhance catalytic activity, and conductivity [16], as described in section 3.2 .

Evidently, the electrochemical biosensor amplifies the signals received from the tumour markers. Figure 7 [16] provides a visual representation of how a biosensor records and amplifies these signals. It is noted that the electrochemical signals that are recorded are directly proportional to the sandwich-like system on the electrode surface. Due to the properties of GNPs and the loading of anti-free PSA antibodies, specific recognition, the antibody's high binding affinity, and the selectivity and sensitivity of the sensor are improved [16]. Figure 8 [16] provides a sideby-side comparison of the GNP/graphene oxide electrochemical biosensor discussed in this paper. The results stem from the analysis of six patients where these graphs indicate that selectivity is an important characteristic to consider in biosensors.

\section{PIEZOELECTRIC BIOSENSORS}

Generally, piezoelectric biosensors are capable of measuring the mass changes as a result of the biological recognition process. The principle by which this biosensor functions, stems from the relation between mechanical stress and an electrical charge in a solid, first discovered in 1880 by the Curie brothers. It was concluded that when mechanical stress is applied to a crystal, there are electrical changes present with a voltage that is proportional to the stress [3, 17]. There is a diverse range of materials that showcase the piezoelectric principle such as quartz ( $\mathrm{SiO} 2)$ due to the quartz crystal microbalance (QCM).

Since GNPs are high in density as well as surface-volume ratios, they can amplify mass changes within crystals, research is being conducted on coupling GNPs with the QCM process to improve analytical sensitivity [3]. 


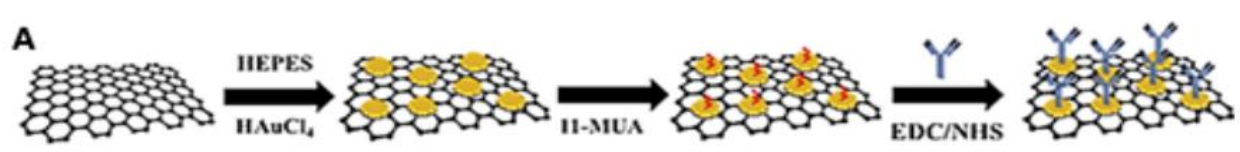

B

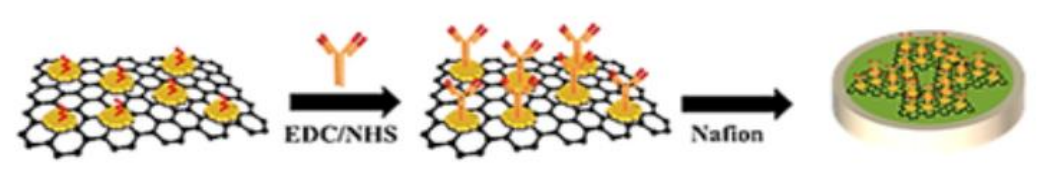

C
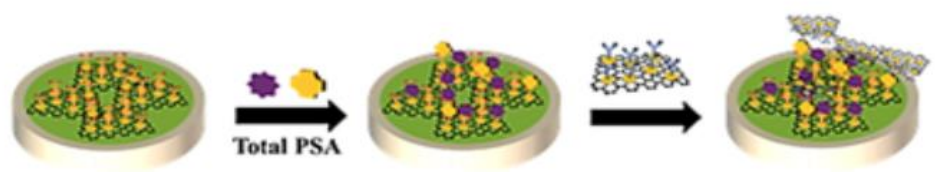
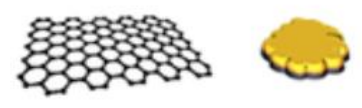

Graphene

Gold

Oxide

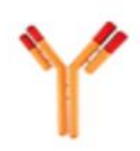

Anti-

Total PSA

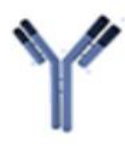

Anti-
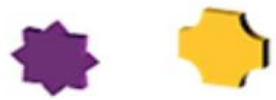

Total

PSA
Free

PSA

Figure 7: (A) Synthesis of graphene oxide, GNPs and anti-total PSA antibody; (B) anti-total PSA antibody is attached to the surface of the electrode of the biosensor to capture antigens; (C) Following a sandwich-like system, the resulting product from (B) is incubated with graphene oxide and GNPs [16].
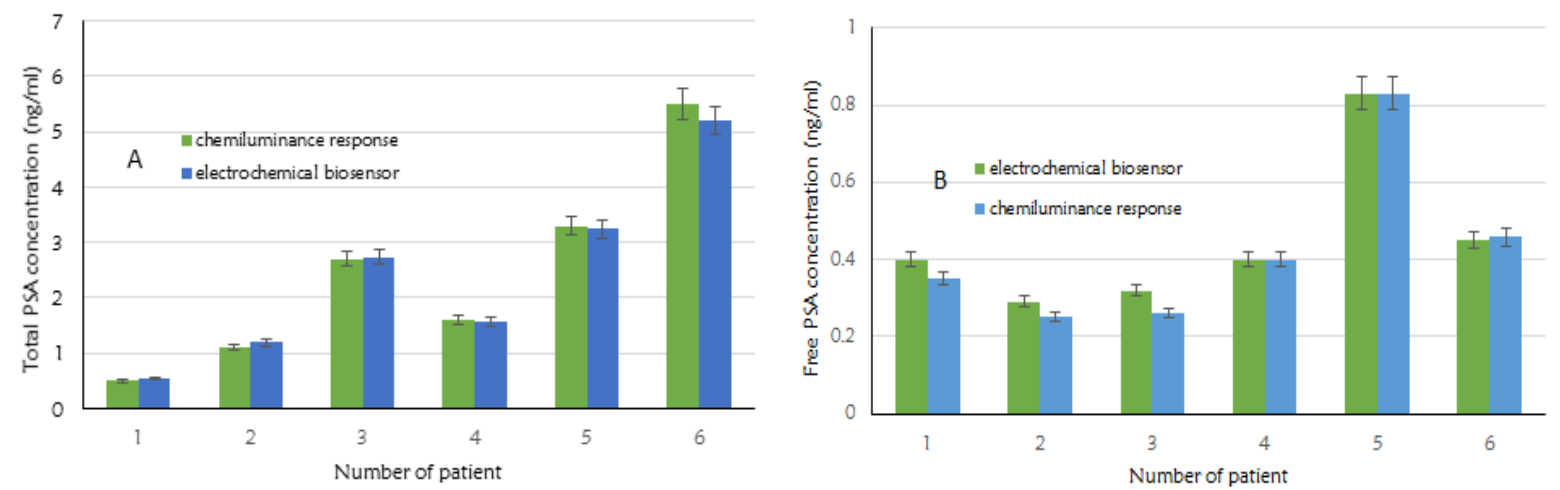

Figure 8: Comparison of (A) total and (B) free PSA concentration deduction results obtained by electrochemical sensor [16].

\subsection{Functionality}

As mentioned above, piezoelectric biosensors are mass-sensitive devices and GNPs increase the analytical sensitivity of these devices through the amplification of mass change [3]. It is important to note that the process of a material under mechanical stress produces a voltage, and also works in the opposite direction (a voltage given to the surface of material causes mechanical stress). In biosensors, the alternating voltage is given on a surface by two electrodes. The alternating voltage produces mechanical oscillations of the material and the frequency of the oscillations is measured and recorded as the oscillation circuit processes. The change in frequency is a result of the analyte on the surface of the electrodes (on the crystal) [18].

Regarding biosensors specifically, the piezoelectric effect is an ideal platform for the development of these devices. It can record affinity interactions independently through the sensitivity in micrograms that lead to evident changes in oscillations. The principle of affinity interaction is defined as the strength of binding between a molecule and its ligand (or binding partner) [18]. For example, the binding interaction between a protein and an inhibitor.

These principles are essential to the piezoelectric effect and development of piezoelectric biosensors [18]. In regard to GNP-based piezoelectric biosensors, the nanoparticle's ability to enhance the sensitivity of the mechanisms leads to surface and mass enhancement effects [3]. In conclusion, the sensitivity and regeneration ability of biological recognition elements on the electrode surface of these biosensors is determined by the quantity and activity of those elements on the electrode surface [3]. 


\subsection{Properties of GNPs in Piezoelectric Biosensors}

The main properties of gold nanoparticles that are attractive for the improvement of piezoelectric biosensors are as follows: surface area-volume ratio, biocompatibility, and density [3]. Beginning with the first property listed above, the surface area-volume ratio is high in gold nanoparticles. In piezoelectric biosensors, this provides a large number of interaction sites to amplify the sensing surface area, meanwhile maintaining bioactivity. This overall improves the quantity, quality, and activity of biological recognition elements [3]. Due to the incredible biocompatibility of GNPs, the stability of biological recognition in piezoelectric biosensors is greatly improved [3]. This is a result of elemental gold's non-harmful effect on living tissue. Finally, the high-density gold nanoparticles allow for mass amplification. It is generally applied via the interaction between an analyte and the sensing surface of the GNPs.

\subsection{Applications: Piezoelectric Biosensors as Immunosensors}

Piezoelectric biosensors have been developed into immunosensors. They are analytical devices used to determine macromolecular compounds and microorganisms. These biosensors consist of a biorecognition element, which in this case is an antibody. The specificity of the antibody impacts the specificity of the immunosensor as a whole [18]. Generally, the specificity of the immunosensor is indicated by the specificity of the antibody and the electrode, whereas the remaining piezoelectric material will not be sensitive to any non-specific reactions with interfering compounds [18]. The fundamental principle of piezoelectric biosensors indicates that the voltage produced by mechanical stress also works in the opposed situation (mechanical stress produced by a voltage). This same principle applies to immunosensors since the immobilized antibody can recognize antigens, as well as the vice versa, as seen in figure 9 [18]. This is an indication that the device contains an immobilized antigen that can then be used for the recognition of just the antibody as the molecule under analysis, making piezoelectric immunosensors a crucial and attractive tool in diagnosing diseases [18].

The quartz crystal microbalance (QCM) process is used in conjunction with GNPs due to QCM's vast use in electronic devices as well as its commercial availability [18]. An example of GNPs and QCM being used in immunosensing is a dendritic amplification immunoassay using GNPs. A GNP immunocomplex was able to successfully detect low IgG (immunoglobulin G) levels, approximately $3.5 \mathrm{ng} / \mathrm{mL}$ in humans [3]. Using a gold electrode surface of the QCM via a protein, goat anti-human IgC was immobilized. The dendritic amplification process occurs through the interaction of the goat anti-human IgC with IgG-functionalized GNPs. As well, the successive interaction between the immunocomplex of the protein and the IgC altered GNPs [19].

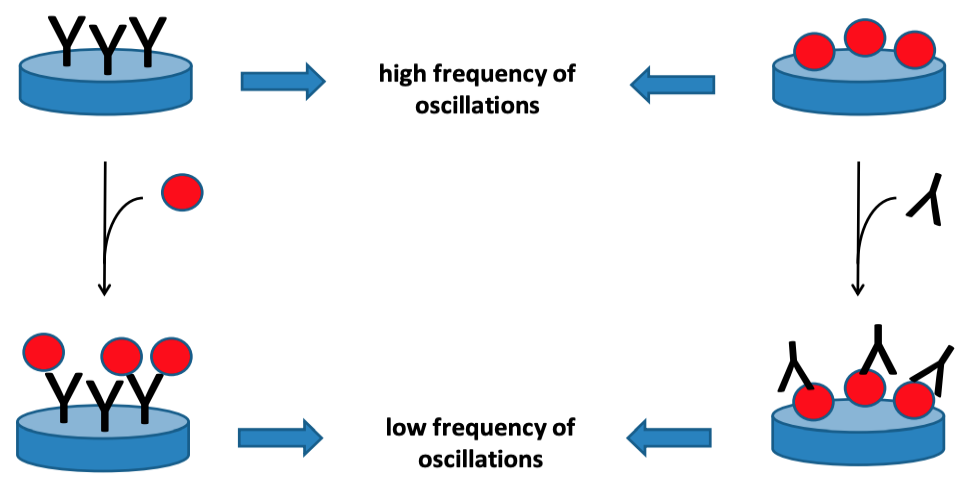

Figure 9: Principle of piezoelectric immunosensors [18].

\section{ADVANTAGES AND DISADVANTAGES}

Optical Biosensors have improved sensitivity for instance in DNA sensors with GNPs responses 1000 times more sensitive than without [20]. Electron transfer rate of 5000 per second with GNPs while 700 per second without GNPs [21]. This speed of transfer is very advantageous and in pairing with the immunity of the signal to either the electrical or magnetic interference makes optical biosensors extremely favourable to be continued to be integrated into biosensing/imaging techniques. The main disadvantage of the use of optical biosensors is the high cost of instrumentation [21]. Within the last decade, there have been great advancements in the use of optical biosensors however, they cannot completely cover the conventional methods of the technology used in many fields such as the biomedical field [21]. Some of these drawbacks include the ability for biomaterial immobilization where the material loss of biomolecules is observed [22] in order. Furthermore, contamination and uniformity of the biosensors is also a drawback where biomolecules and chemicals leak out of the biosensors causing there to create contamination. Moreover, when using optical biosensors, the selection range must be a large selection range in order for the sensor to detect the variety of biomaterials. However, with the substantial use of optical biosensors in not only the biomedical field but also in fields such as environmental monitoring, industrial and food processes, health care, clinical analysis [23], there is an increased array of testing and usage where these drawbacks can be overcome and fixed. 
Electrochemical Biosensors have improved sensitivity and stability for instance in glucose biosensors with GNPs achieving detection limits of 0.18 uM [24]. An NADH sensor based on GNPs shows a $780 \mathrm{mV}$ overpotential decrease without any electron transfer mediators [3]. Furthermore, the electrochemical biosensors require less sample volume and have a cost-effective rapid response output. Moreover, electrochemical biosensors enable regions with limited resources to perform healthcare diagnostics without the need for trained professionals [4]. However, with these advantages, there are some disadvantages such as the sensitivity of the electrochemical biosensors to the sample matrix effects and the lower shelf life of the sensor [4]. Through future research and collaborations with hospitals, there can be greater fundamental use of the electrochemical biosensor when used for improving disease diagnosis and treatment [4]. Piezoelectric biosensors have improved sensitivity for instance in DNA sensors using GNPs as amplification tags with a detection limit [3]. These sensors have the principle detection of identifying changes in mass [3]. In the GNPs, Piezoelectric Biosensors play a vital role in amplifying specific changes in mass. Furthermore, these biosensors are high in density, have a large surface volume ratio and are biocompatible. The advantages of these sensors are that they contain an improved sensitivity, as listed in Table 1 [3].

Table 1: Summary of the GNP based biosensors [3]

\begin{tabular}{|c|c|c|c|c|c|}
\hline Biosensor & Principle of detection & Role of GNPs & Properties of GNPs & Advantages & Application \\
\hline $\begin{array}{l}\text { Optical } \\
\text { Biosensors }\end{array}$ & $\begin{array}{l}\text { Changes in optical } \\
\text { (light) properties }\end{array}$ & $\begin{array}{l}\text { Enhancement of } \\
\text { refractive index changes }\end{array}$ & $\begin{array}{l}\text { Larger dielectric } \\
\text { constant, high density }\end{array}$ & $\begin{array}{l}\text { Improved } \\
\text { sensitivity }\end{array}$ & $\begin{array}{l}\text { Colorimetric } \\
\text { Bioassays }\end{array}$ \\
\hline $\begin{array}{l}\text { Electrochemica } \\
\text { I Biosensors }\end{array}$ & $\begin{array}{l}\text { Changes in electrical } \\
\text { characteristics }\end{array}$ & $\begin{array}{l}\text { “Electron wires", } \\
\text { catalysis, immobilization }\end{array}$ & $\begin{array}{l}\text { Large area-volume } \\
\text { ratio, biocompatibility, } \\
\text { high surface energy, } \\
\text { conductivity, quantum } \\
\text { dimension, interface- } \\
\text { dominated properties, } \\
\text { large surface area }\end{array}$ & $\begin{array}{l}\text { Improved } \\
\text { sensitivity, } \\
\text { selectivity, } \\
\text { stability }\end{array}$ & $\begin{array}{l}\text { Tumour } \\
\text { Biomarker } \\
\text { Detection }\end{array}$ \\
\hline $\begin{array}{l}\text { Piezoelectric } \\
\text { Biosensors }\end{array}$ & Changes in mass & $\begin{array}{l}\text { Amplification of changes } \\
\text { in mass }\end{array}$ & $\begin{array}{l}\text { High density, large } \\
\text { surface-volume ratio, } \\
\text { biocompatibility, high } \\
\text { density }\end{array}$ & $\begin{array}{l}\text { Improved } \\
\text { sensitivity }\end{array}$ & $\begin{array}{l}\text { Immunosenso } \\
\text { rs }\end{array}$ \\
\hline
\end{tabular}

\section{RECOMMENDATIONS}

Gold nanoparticle-based biosensors present promising opportunities for advancing and developing analytical systems in many clinical and non-clinical applications. This is due to the unique properties of GNPs that show a variety of superior analytical behaviours. This review has demonstrated the many advantages of GNPs for the three most common types of biosensors, as well as the role in which the GNPs play in the process of biosensing discussed in terms of sensitivity, selectivity, reliability, etc. There are many implementations of GNP-based biosensors in different sectors. The following is a list of recent advances and future applications:

1. Intracellular microRNA Quantification [25].

a. Mass-sensitive microRNA sensing surface uses a probe, established by gold-nanoparticles.

b. The sensing method is to pick up on the frequency shift of QCM (quartz crystal microbalance) sensors (Piezoelectric biosensors concept).

c. QCM sensors are compatible with different operating conditions (gaseous or liquid mediums) [26] making it a useful technique in health care.

d. Gold-nanoparticles amplify the signal while coupled with an enzymatic amplification process. The sensor detects microRNA-203 in MCF-7 cells (typically seen in tumour growth) with exceptional sensitivity and selectivity.

2. Localized Surface Plasmon Field - Fibre-Optic Biosensor [27].

a. The benefits of gold-nanoparticles for localized plasmon resonance (LSPR) optical sensors is the depth of penetration of surface plasmons - affecting the sensitivity.

b. The size of the GNP affects the penetration depth of the LSPR biosensor.

c. Ultimately, the greatest diameter exhibited the greatest penetration depth - indicating that the size of the GNP directly impacts the functionality of the LSPR sensor.

3. Enhancement of Direct Electron Transfer of Glucose Oxidase [28].

a. Gold nanoparticles are used with titanite nanotubes (TNT). TNT aids in the direct electron transfer of glucose oxidase.

b. Combined with the GNP-TNT nanocomposite is an ionic liquid (bromated 1-decyl-3-methyl imidazole) to immobilize the structure and further promote the electron transfer between glucose oxidase and the electrode. Volumetric results showed that there is a strong electrocatalytic capability towards glucose electron transfer rate constant of $7.1 \mathrm{~s}-1$ at $180 \mathrm{mVs}-1$. 
c. High sensitivity in the calibration for glucose - 5.1A mM-1.

d. The measured parameters indicate superior analytical performance of the biosensor - compared to those developed with other nanoparticles. As well, increased biocompatibility and electrical conductivity of the nanocomposite became evident.

4. Microfluidic-based Biosensor [29].

a. One of the fastest-growing technologies among biosensors.

b. Gold nanoparticles play the main role in their development in order to promote biomolecular detection with higher sensitivity and effectiveness.

5. Gold-Nanoparticles for Biosensors in Healthcare [30].

a. Scientists define an Elite Group of Nanomaterials, including Graphene, CNTs (Carbon Nanotubes), ZnO, and Gold. These nanomaterials are used in the modification of electrodes for Electrochemical Sensing of Analytes.

b. In addition to healthcare, these biosensors show promise for on-site detection of explosives.

c. Development of genosensor, immunosensor, and enzymatic biosensors are in progress using the Elite Group of Nanomaterials. Gold shows the greatest promise in the detection of immunological molecules and is more commonly used in immunological sensing.

d. The best results are shown when a combination of these materials is used with the electrodes. This promotes great stability, reproducibility, and sensitivity, and signal amplification as a result of electrochemical and electrocatalytic properties of these materials.

e. Detection of glucose, lgG, IgE, sequence-specific DNA, amino acids, viruses and bacteria.

6. Plasmonic Biosensors [14]

a. GNPs allow for the altercation of size, shape, composition, coupling of intense colours under light, enhance optical signals (i.e. fluorescence and Raman scattering, and improve plasmonic properties in specific optical systems.

b. Patterning GNPs on electrodes (LSPR).

c. Controlling gap distance between particles for SERS-based biosensor.

d. In a fluorescent signal, controlling the distance between a GNP and a fluorophore. As well as the control of the dispersion stability in the development of colorimetric biosensors.

e. Used in the detection of cancer biomarkers [31].

\section{CONCLUSIONS}

1. Gold colloids are becoming a key component to many industries such as health care, food, engineering, etc.

2. Gold particles are used in immunochemistry, photothermal therapy agents and detection of cancerous cells these properties are now being applied to engineering and biosensing applications.

3. Biosensors are becoming increasingly relevant in the food industry to ensure quality and safety. As well as the fermentation industry for the detection of glucose concentrations, and metabolic engineering in the monitoring of cellular metabolism. In general, the use of biosensors has diverse applications due to their diverse functionality.

4. Gold nano-particle properties include biocompatibility, conductivity, catalytic properties, high surface-tovolume ratio, and high density. This makes the nanomaterial grow exceptionally in the bioassay field.

5. It was discussed how these distinct properties can make each kind of biosensor most effective for specific applications, such as colorimetric bioassays, tumour biomarker detection, and immunosensors.

6. The field of research around GNP-based biosensors is still in its infancy in terms of real-world applications, as widespread use is not currently possible since there remain challenges to be addressed before the full potential of applications can be realized. In order to fully exploit the potential applications of GNP-based biosensors, the following research areas should be given attention:

a. Design of GNP-based biosensors for high throughput and multiplexed identification of biomarkers.

b. Production of GNPs with long-term sustainability in a variety of environments.

c. Nanostructure and size of the GNPs, as this affects various optical and electrical properties.

d. Improvement for design and synthesis of GNPs with better-defined geometrical properties that will promote more applicable uses.

e. Compositing other nanomaterials with GNPs; hybrids of other nanomaterials in combination with GNPs have the potential to acquire more distinct, and useful properties.

f. Improvement for the analytical performance of GNPs at the present stage (e.g. preventing non-specific adsorption of biomolecules or shortening the length of analysis time).

\section{REFERENCES}

1. B. Barua, T. Jackson, and N. Esmail, "The Effect of Wait Times on Mortality in Canada," Fraser Institute, May2014. [Online]. Available: https://www.fraserinstitute.org/. [Accessed: 19-Apr-2021].

2. P. Mohankumar, J. Ajayan, T. Mohanraj, and R. Yasodharan, "Recent developments in biosensors for healthcare and biomedical applications: A review," ResearchGate, Jul-2020. [Online]. Available: 
https://www.researchgate.net/publication/343356920_Recent_developments_in_biosensors_for_healthcare_an d biomedical applications A review. [Accessed: 19-Apr-2021].

3. Y. Li, H. J. Schluesener, and S. Xu, "Gold nanoparticle-based biosensors," SpringerLink, Mar-2010. [Online]. Available: https://link.springer.com/article/10.1007/BF03214964. [Accessed: 19-Apr-2021].

4. G. Ghosh, "Early detection of cancer: Focus on antibody coated metal and magnetic nanoparticle-based biosensors," Sensors International, [Online]. Available: https://www.sciencedirect.com/science/article/pii/S2666351120300504. [Accessed: 19-Nov-2021].

5. L. C. Clark and C. Lyons, "Electrode systems for continuous monitoring in cardiovascular surgery," Annals of the New York Academy of Sciences, 31-Oct-1962. [Online]. Available: https://pubmed.ncbi.nlm.nih.gov/14021529/. [Accessed: 19-Apr-2021].

6. V.-D. T and B. Callum, "Biosensors and biochips: Advances in biological and Medical Diagnostics," Fresenius' journal of analytical chemistry, Mar-2000. [Online]. Available: https://pubmed.ncbi.nlm.nih.gov/11225766/. [Accessed: 19-Apr-2021].

7. D. A. Giljohann, D. S. Seferos, W. L. Daniel, M. D. Massich, P. C. Patel, and C. A. Mirkin, "Gold nanoparticles for biology and medicine," Angewandte Chemie (International ed. in English), 26-Apr-2010. [Online]. Available: https://pubmed.ncbi.nlm.nih.gov/20401880/. [Accessed: 19-Apr-2021].

8. H. Liu, J. Ge, E. Ma, and L. Yang, “Advanced biomaterials for Biosensor and theranostics," ResearchGate, Jan$2019 . \quad$ [Online]. Available: https://www.researchgate.net/publication/330342204_Advanced_biomaterials_for_biosensor_and_theranostic s. [Accessed: 19-Apr-2021].

9. A. Mandal, "Biosensor applications," News, 26-Feb-2019. [Online]. Available: https://www.newsmedical.net/health/Biosensor-Applications.aspx. [Accessed: 19-Apr-2021].

10. S. Das, S. Mitra, S. M. P. Khurana, and N. Debnath, "Nanomaterials for Biomedical Applications," Taylor \& Francis, 23-Jan-2014. [Online]. Available: https://www.tandfonline.com/doi/full/10.1080/21553769.2013.869510. [Accessed: 19-Apr-2021].

11. P. Jiang, Y. Wang, L. Zhao, C. Ji, D. Chen, and L. Nie, "Applications of gold nanoparticles in non-optical biosensors," Nanomaterials (Basel, Switzerland), 26-Nov-2018. [Online]. Available: https://www.ncbi.nlm.nih.gov/pmc/ahttps://www.ncbi.nlm.nih.gov/pmc/articles/PMC6315477/rticles/PMC63 15477/. [Accessed: 19-Nov-2021].

12. P. Damborský, J. Švitel , and J. Katrlík, “Optical biosensors,” Essays in biochemistry, 30-Jun-2016. [Online]. Available: https://pubmed.ncbi.nlm.nih.gov/27365039/. [Accessed: 19-Apr-2021].

13. C. J. Murphy, A. M. Gole, S. E. Hunyadi, J. W. Stone, P. N. Sisco, A. Alkilany, B. E. Kinard, and P. Hankins, "Chemical Sensing and imaging with metallic nanorods," University of Illinois Urbana-Champaign, 19-Jun-2015. [Online]. Available: https://experts.illinois.edu/en/publications/chemical-sensing-and-imaging-with-metallicnanorods. [Accessed: 19-Apr-2021].

14. J.-H. Lee, H.-Y. Cho, H. K. Choi, J.-Y. Lee, and J.-W. Choi, "Application of gold nanoparticle to Plasmonic Biosensors," International journal of molecular sciences, 11-Jul-2018. [Online]. Available: https://pubmed.ncbi.nlm.nih.gov/29997363/. [Accessed: 19-Apr-2021].

15. P. Si, N. Razmi, O. Nur, S. Solanki, C. M. Pandey, R. K. Gupta, B. D. Malhotra, M. Willander, and A. de la Zerda, "Gold nanomaterials for optical biosensing and bioimaging," Nanoscale Advances, 14-Apr-2021. [Online]. Available: https://pubs.rsc.org/en/content/articlelanding/2021/na/dOna00961j. [Accessed: 19-Nov-2021].

16. Z. Akbari Jonous, J. S. Shayeh, F. Yazdian, A. Yadegari, M. Hashemi, and M. Omidi, "An electrochemical biosensor for prostate cancer biomarker detection using graphene oxide-gold nanostructures," Engineering in life sciences, 07-Feb-2019. [Online]. Available: https://www.ncbi.nlm.nih.gov/pmc/articles/PMC6999480/. [Accessed: 19-Apr-2021].

17. P. Mehrotra, "Biosensors and their applications - A Review," Journal of oral biology and craniofacial research, 06-Jan-2016. [Online]. Available: https://www.ncbi.nlm.nih.gov/pmc/articles/PMC4862100/. [Accessed: 19Apr-2021].

18. M. Pohanka, "Overview of piezoelectric biosensors, immunosensors and DNA sensors and their applications," Materials (Basel,Switzerland), 19-Mar-2018.[Online]. Available: https://www.ncbi.nlm.nih.gov/pmc/articles/PMC5873027/. [Accessed: 19-Apr-2021].

19. X. Chu, Z.-L. Zhao, G.-L. Shen, and R.-Q. Yu, "Quartz crystal microbalance immunoassay with dendritic amplification using colloidal gold immunocomplex," Sensors and Actuators B: Chemical, vol. 114, no. 2, pp. 696-704, Aug. 2005.

20. L. He, M. D. Musick, S. R. Nicewarner, F. G. Salinas, S. J. Benkovic, M. J. Natan, and C. D. Keating, "Colloidal au-enhanced surface plasmon resonance for ultrasensitive detection of DNA hybridization," ACS Publications, 08-Sep-2000. [Online]. Available: https://pubs.acs.org/doi/10.1021/ja001215b. [Accessed: 19-Apr-2021].

21. Y. Weizmann, F. Patolsky, E. Katz, and I. Willner, "Amplified DNA sensing and immunosensing by the rotation of functional magnetic particles," ACS Publications, 04-Mar-2003. [Online]. Available: https://pubs.acs.org/doi/10.1021/ja028850x. [Accessed: 19-Apr-2021]. 
22. D. Dey and T. Goswami, "Optical biosensors: A revolution towards Quantum Nanoscale Electronics Device Fabrication," Journal of biomedicine \& biotechnology, 29-Oct-2011. [Online]. Available: https://pubmed.ncbi.nlm.nih.gov/22131802/. [Accessed: 19-Apr-2021].

23. S. A. Hussain, P. K. Paul, D. Dey, D. Bhattacharjee, and S. Sinha, "Immobilization of single strand DNA on solid substrate," Chemical Physics Letters, vol. 450, no. 1-3, pp. 49-54, Nov. 2007.

24. D. Branton, D. W. Deamer, A. Marziali, H. Bayley, S. A. Benner, T. Butler, M. Di Ventra, S. Garaj, A. Hibbs, X. Huang, S. B. Jovanovich, P. S. Krstic, S. Lindsay, X. S. Ling, C. H. Mastrangelo, A. Meller, J. S. Oliver, Y. V. Pershin, J. M. Ramsey, R. Riehn, G. V. Soni, V. Tabard-Cossa, M. Wanunu, M. Wiggin, and J. A. Schloss, "The potential and challenges of Nanopore sequencing," Nature biotechnology, 26-Oct-2008. [Online]. Available: https://pubmed.ncbi.nlm.nih.gov/18846088/. [Accessed: 19-Apr-2021].

25. S. Andreescu and L. A. Luck, "Studies of the binding and signaling of surface-immobilized periplasmic glucose receptors on gold nanoparticles: A glucose biosensor application," Analytical Biochemistry, vol. 375, no. 2, pp. 282-290, Jan. 2008.

26. Y. Guo, Y. Wang, G. Yang, J.-J. Xu, and H.-Y. Chen, "MicroRNA-mediated signal amplification coupled with GNP/dendrimers on a mass-sensitive biosensor and its applications in intracellular microrna quantification," Biosensors \& bioelectronics, 15-Nov-2016. [Online]. Available: https://pubmed.ncbi.nlm.nih.gov/27311115/. [Accessed: 19-Apr-2021].

27. A. Alassi, M. Benammar, and D. Brett, "Quartz Crystal Microbalance electronic interfacing systems: A Review," Sensors (Basel, Switzerland), 05-Dec-2017. [Online]. Available: https://pubmed.ncbi.nlm.nih.gov/29206212/. [Accessed: 19-Apr-2021].

28. R. Bharadwaj, S. Mukherji, and S. Mukherji, "Probing the localized surface plasmon field of a gold ...," ResearchGate, Jun-2016. [Online].Available: https://www.researchgate.net/publication/282796834_Probing_the_Localized_Surface_Plasmon_Field_of_a_G old_Nanoparticle-Based_Fibre_Optic_Biosensor. [Accessed: 19-Apr-2021].

29. R. Zhao, X. Liu, J. Zhang, J. Zhu, and D. K. Y. Wong, "Enhancing direct electron transfer of glucose oxidase using a gold nanoparticle: Titanate nanotube nanocomposite on a Biosensor," Macquarie University, 16-Jan-2017. [Online]. Available: https://researchers.mq.edu.au/en/publications/enhancing-direct-electron-transfer-ofglucose-oxidase-using-a-gol. [Accessed: 19-Apr-2021].

30. T. Lakshmipriya, U. Hashim, S. C. B. Gopinath, and N. Azizah, "Microfluidic-based biosensor: Signal enhancement by Gold Nanoparticle," Microsystem Technologies, 16-Jul-2016. [Online]. Available: https://link.springer.com/article/10.1007/s00542-016-3074-1. [Accessed: 19-Apr-2021].

31. S. Kumar, W. Ahlawat, R. Kumar, and N. Dilbaghi, "Graphene, carbon nanotubes, zinc oxide and gold as elite nanomaterials for fabrication of biosensors for Healthcare," Biosensors \& bioelectronics, 15-Aug-2015. [Online]. Available: https://pubmed.ncbi.nlm.nih.gov/25899923/. [Accessed: 19-Apr-2021].

32. L. Xiao, A. Zhu, Q. Xu, Y. Chen, J. Xu, and J. Weng, "Colorimetric biosensor for detection of cancer biomarker by AU nanoparticle-decorated bi 2 se 3 nanosheets," ACS applied materials \& interfaces, 06-Feb-2017. [Online]. Available: https://pubmed.ncbi.nlm.nih.gov/28164701/. [Accessed: 19-Apr-2021]. 